ISAHP Article: Masaaki Shinohara /DAP FOR INCOMPLETE INFORMATION CASE

International Symposium of the Analytic Hierarchy Process 2014, Washington D.C., U.S.A.

\title{
DYNAMIC AVERAGING PROCESS FOR INCOMPLETE INFORMATION CASE
}

\author{
Masaaki Shinohara* \\ Nihon University \\ Izumi-chou, Narashino \\ Chiba 275-8575, Japan
}

\begin{abstract}
Dynamic Averaging Process is studied for the incomplete information case where some elements in a pairwise comparison matrix are missing. As the averaging operation of a DAP, the generalized mean with the $p$-th power, or the $p$-th power mean, is considered. Note that those with $p=1, p=-1, p \rightarrow 0$, and $p \rightarrow+\infty$ are the arithmetic mean, the harmonic mean, the geometric mean and the maximum operation, respectively.

First, we prove that the convergent weight vector of a geometric DAP, or the DAP with geometric mean averaging (the power mean with $p \rightarrow 0$ ), is equal to the LLS(Logarithmic Least Square) solution under the reciprocity condition $\left(a_{i j \times} a_{j i}=1\right)$, the self-identity $\operatorname{condition}\left(a_{i i}=1\right)$,etc.

Second, the Harker method is modified in two ways so that it can cope with the $p$-th power mean and it can take into account the effect of the eigenvector $\lambda$, or the stationary state rate.

Third, we prove that the convergent weight vector of a $p$-th power DAP, or a DAP with the $p$ th power averaging(power mean with $p \neq 0$ ), is equal to the convergent weight vector of the modified Harker method under the stationarity condition $(\boldsymbol{x}(t)=\lambda \boldsymbol{x}(t-1)$, or the existence of the stationary rate $\lambda$ ).

Finally, since a DAP can be regarded as the power method for calculating an eigenvector for the generalized algebraic system, the possibility of using the geometric DAP, or the LLS, is discussed to estimate the weight vector for the incomplete information case.
\end{abstract}

Keywords: incomplete information, eigenvector, dynamic averaging process, Harker method, Logarithmic Least Square solution, $p$-th power mean, geometric mean

\section{Introduction}

The dynamic averaging process or DAP, which is supposed to simulate the priority weight determination process on the cognitive layer in a human mind, is studied for pairwise comparison matrixes where some elements are missing. Some elements in a comparison matrix are missing because they cannot be measured due to technical reasons and they can be intentionally eliminated in order to decrease the burden of pairwise comparisons, especially in a large-scale comparison matrix. For these incomplete information cases, the Logarithmic Least Square (LLS) method, Harker method (Harker, 1987), the method of assuming and updating missing elements, and other methods, have been applied to estimate the priority weight vector. Other than the se conventional methods, another method can be conceived by directly applying the dynamic averaging process (DAP), as presented in Chapter 2 of this paper.

In Chapter 2, we present the DAP updating formula for the case of incomplete information and the $p$-th power mean averaging. In Chapter 3, we prove that the

Corresponding author

International Symposium of

the Analytic Hierarchy

Washington, D.C.

Process June 29 - July 2, 2014 
convergent weight vector of a geometric DAP, or the DAP with geometric mean averaging (the power mean with $p \rightarrow 0$ ), is equal to the LLS(Logarithmic Least Square) solution under the reciprocity condition $\left(a_{i j} \times a_{j i}=1\right)$, the self-identity condition (

$\left.a_{i i}=1\right)$ and the stable stationarity condition $(\boldsymbol{x}(t)=\lambda \boldsymbol{x}(t-1)$ with $\lambda=1)$ of the geometric DAP. In Chapter 4, the Harker method is modified in two ways so that it can cope with the $p$-th power mean and it can take into account the effect of the eigenvector $\lambda$, or the stationary state increasing (or decreasing) rate. In Chapter 5, we prove that the convergent weight vector of the $p$-th power DAP, or the DAP with the $p$-th power averaging (power mean with $p \neq 0$ ), is equal to the convergent weight vector of the modified Harker method under the stationarity condition $(\boldsymbol{x}(t)=\lambda \boldsymbol{x}(t-1))$, or the existence of the stationary rate $\lambda$ ).

\section{Incomplete-information DAP updating formula for the p-th power mean averaging}

The DAP updating formula for the case of incomplete information and the $p$-th power mean averaging is obtained as in (1) or (2).

Time-invariant case: $w_{k}(t+1)=\left(\frac{1}{\left|s_{k}\right|} \sum_{j \in S_{k}}\left(a_{k j} w_{j}(t)\right)^{p}\right)^{1 / p}(\mathrm{k}=1,2, \ldots, \mathrm{N})$

Time-variant case: $w_{k}(t+1)=\left(\frac{1}{\left|S_{k}(t)\right|} \sum_{j \in S_{k}(t)}\left(a_{k j}(t) w_{j}(t)\right)^{p}\right)^{1 / p}(\mathrm{k}=1,2, \ldots, \mathrm{N})$

$S_{k}$ : the set of items which are directly compared with item $k$, or the set of $j$ 's whose ${ }^{a_{k j}}$ 's are not missing in the $k$-th row of an $N \times N$ comparison matrix $\mathrm{A}=\left\{a_{k j}\right\}$.

$w_{j}(t)$ : the priority weight of item $j$ at time $t$.

Note that the $p$-th power mean DAP updating formula (1) results in the arithmetic mean DAP as shown by (3) when $p=1$, the harmonic mean DAP as shown by (4) when $p=-1$, the geometric mean DAP as shown by (5) when $p \rightarrow 0$, the max-DAP(Shinohara, 2013)as shown by (6) when $p \rightarrow+\infty$, and so on.

Arithmetic DAP: ${ }^{w_{k}}(t+1)=\frac{1}{\left|s_{k}\right|} \sum_{j \in s_{k}} a_{k j} w_{j}(t) \quad k=1,2, \ldots, \mathrm{N}$

Harmonic DAP: $w_{k}(t+1)=\left(\frac{1}{\left|s_{k}\right|} \sum_{j \in S_{k}}\left(a_{k j} w_{j}(t)\right)^{-1}\right)^{-1}$

Geometric DAP: $w_{k}(t+1)=\left(\prod_{j \in S_{k}} a_{k j} w_{j}(t)\right)^{1 /\left|s_{k}\right|}$

MAX-DAP: $w_{k}(t+1) \_\max _{j \in s_{k}}\left\{a_{k j} w_{j}(t)\right\}$

\section{Geometric DAP convergent solution = LLS solution}

A mathematical proof for "geometric DAP convergent solution = LLS solution" will be given next, using the geometric mean topological formula obtained in the topological researches on the LLS solution (Shinohara, Osawa \& Shinohara, 2005, Mogi \& 
Shinohara, 2011, Shinohara \& Mogi, 2012), where the geometric mean formula (7) has been shown to hold under the reciprocity condition and the self-identity condition.

$w_{k}=\left(\prod_{j \in S_{k}} a_{k j} \cdot \prod_{j \in M_{k}} \frac{w_{k}}{w_{j}}\right)^{\frac{1}{N}}$

$S_{k}$ : the set of non-missing elements in the $k$-th row of comparison matrix A,

$M_{k}$ : the set of missing elements in the $k$-th row of comparison matrix A,

and $\left|S_{k}\right|+\left|M_{k}\right|=N(8)$.

$w_{j}$ : the (estimated) priority weight of item $\mathrm{j}$.

Taking the $N$-th power to the both sides of (7), (9) is obtained.

$w_{k}{ }^{N}=\prod_{j \in S_{k}} a_{k j} \cdot \prod_{j \in M_{k}} \frac{w_{k}}{w_{j}}$

Dividing the both sides of (9) by $w_{k}^{\left|M_{k}\right|},(10)$ is obtained.

$w_{k}^{N-\left|M_{k}\right|}=\prod_{j \in S_{k}} a_{k j} \cdot \prod_{j \in M_{k}} \frac{1}{w_{j}}$

Considering that the weight vector $\mathrm{w}$ has the degree of freedom by a constant factor, we can assume (11), and (10) can be transformed into (12).

$\prod_{j=1, \ldots, N} w_{j=1}$

$w_{k}{ }^{N-\left|M_{k}\right|} \prod_{j \in S_{k}} a_{k j} \cdot \prod_{j \in M_{k}} \frac{1}{w_{j}} \cdot \prod_{j=1, \ldots, N} w_{j=} \prod_{j \in S_{k}} a_{k j} w_{j}$

Taking the $1\left(\mathrm{~N}-\left|M_{k}\right|\right)_{-t h}$ power to the both sides of (12), (13) is obtained.

$w_{k}=\left(\prod_{j \in S_{k}} a_{k j} w_{j}\right)^{\frac{1}{\left(\mathrm{~N}-\left|M_{k}\right|\right)}}=\left(\prod_{j \in S_{k}} a_{k j} w_{j}\right)^{\frac{1}{\left(S_{k} \mid\right)}}$

This formula (13) coincides with the geometric DAP updating formula (5), if the stable stationarity condition (14) holds in (5).

$w_{j}(t+1)_{=} w_{j}(t)=w_{j} \quad(j=1, \ldots, N)$

\section{Harker method and modified Harker method}

The Harker method is modified so that the method gives a convergent weight vector which is equal to the convergent weight vector of the $p$-th power mean DAP. The original Harker method insists to make the complete-information Harker matrix $H=\left\{h_{k j}\right\}$ from an incomplete-information comparison matrix $\mathrm{A}=\left\{a_{k j}\right\}$ as follows:

$h_{i i}=a_{i i+}\left|M_{i}\right|, \mathrm{i}=1, \ldots, \mathrm{N}$

$h_{k j}=\left\{\begin{array}{c}0 \text { if } a_{k j} \text { is missing. } \\ a_{k j} \text { if } a_{k j} \text { is not missing. }\end{array}\right.$ 
In order to take the $p$-th power mean DAP into consideration $(p \neq 0)$, the original Harker method is modified as follows:

[Case of $p>0$ ]

$h_{i i}=\left(a_{i i}+\lambda^{p}\left|M_{i}\right|\right)^{\frac{1}{p}}$

$h_{k j}=\left\{\begin{array}{c}0 \text { if } a_{k j} \text { is missing. } \\ a_{k j} \text { if } a_{k j} \text { is not missing. }\end{array}\right.$

[Case of $p<0$ ]

$h_{i i}=\left(a_{i i}+\lambda^{p}\left|M_{i}\right|\right)^{\frac{1}{p}}$

$h_{k j}=\left\{\begin{array}{c}+\infty \text { if } a_{k j} \text { is missing. } \\ a_{k j} \text { if } a_{k j} \text { is not missing. }\end{array}\right.$

$\lambda$ : stationary state increasing(or decreasing) rate of a raw DAP performance, $\left|M_{i}\right|$ : the number of missing elements in the $i$-th row.

\section{Convergent solution of the $p$-th power mean DAP = convergent solution of the modified Harker method}

A mathematical proof for "convergent solution of the $p$-th power mean DAP $=$ convergent solution of the $p$-th power mean modified Harker method" will be given next, assuming the existence of a stationary state increasing (or decreasing) rate $\lambda$ of a raw DAP performance.

The updating formula for the $p$-th power mean DAP is shown by (21), directly from (1) in Chapter 1.

$w_{k(\mathrm{t}+1)}=\left(\frac{1}{\left|s_{k}\right|} \sum_{j \in S_{k}}\left(a_{k j} w_{j}(t)\right)^{p}\right)^{\frac{1}{p}} \quad(\mathrm{k}=1,2, \ldots, \mathrm{N})$

Taking the $p$-th power to the both sides of (21), (22) is obtained.

$\left.w_{k}(t+1)^{p}=\frac{1}{\left|s_{k}\right|} \sum_{j \in s_{k}}\left(a_{k j} w_{j}(t)\right)^{p}\right)$

Multiplying $\left|S_{k}\right|$ and then adding $\left|M_{k}\right| w_{k}(\mathrm{t}+1)^{p}$ to the both sides of (22), (23) is obtained.

$\left|S_{k}\right| w_{k}(\mathrm{t}+1)^{p}{ }_{+}\left|M_{k}\right| w_{k}(\mathrm{t}+1)^{p}=\sum_{j \in S_{k}}\left(a_{k j} w_{j}(t)\right)^{p}+\left|M_{k}\right| w_{k}(\mathrm{t}+1)^{p}$

Assuming the existence of a stationary state rate $\lambda$, or that (24) holds for a sufficiently large $t,(23)$ is transformed into (25).

$w_{k}(\mathrm{t}+1)=\lambda w_{k}(\mathrm{t}) \quad(\mathrm{k}=1,2, \ldots, \mathrm{N})$
$N w_{k}(t+1)^{p} \sum_{j \in S_{k}}\left(a_{k j} w_{j}(t)\right)^{p}{ }_{+} \lambda^{p}\left|M_{k}\right| w_{k}(t)^{p}$ 
Assuming $k \in S_{k},(25)$ is rearranged as follows.

$N w_{k}(t+1)^{p}=\sum_{j \in S_{k}-\{k\}}\left(a_{k j} w_{j}(t)\right)^{p}+\left(a_{k k}{ }^{p}+\lambda^{p}\left|M_{k}\right|\right) w_{k}(t)^{p}$

$=\sum_{j \in S_{k}-\{k\}}\left(a_{k j} w_{j}(t)\right)^{p}+\left[\left(a_{k k}^{p}+\lambda^{p}\left|M_{k}\right|\right)^{\frac{1}{p}} w_{k}(t)\right]^{p}+\underline{Q}$

Noting that $w_{j}$ is positive finite and that $\left(a_{k j} w_{j}(t)\right)^{p}=\underline{Q}\left(j \in M_{k}\right)$ if $a_{k j=0}$ for $\mathrm{p}>0$ and

$a_{k j}=+\infty$ for $\mathrm{p}<0,(25)$ is finally transformed into (27) with (28), which is nothing but the $p$-th power mean updating formula with the modified Harker matrix $\mathrm{H}$.

$N w_{k}(t+1)^{p} \sum_{j \in S_{k}-\{k\}}\left(a_{k j} w_{j}(t)\right)^{p}{ }_{+}\left[\left(a_{k k}^{p}+\lambda^{p}\left|M_{k}\right|\right)^{\frac{1}{p}} w_{k}(t)\right]^{p} \sum_{j \in M_{k}}\left(a_{k j} w_{j}(t)\right)^{p}$

$a_{k j=}\left\{\begin{array}{ll}0 & p>0 \\ +\infty & \mathrm{p}<0\end{array} \quad\left(j \in M_{k}\right)\right.$

*In case $k \notin S_{k}$, or $\mathrm{k} \in M_{k}$, the original updating formula (21) is transformed into (29), where $a_{k k}$ is defined by (28).

\section{Conclusions}

The dynamic averaging process, or DAP, is studied for the case of incomplete information of pairwise comparison data, and we proved that "geometric DAP convergent solution = LLS solution" and that "convergent solution of the $p$-th power mean DAP = convergent solution of the modified Harker method."

Following are some considerations obtained through this study:

(1) The $a_{k j}$ data belongs to a class of ratio data, without any doubt, but note that the $a_{k j} w_{j}$ data, or the $w_{j}$ data can also be regarded to belong to another class of ratio data, because the $w_{j}$ data has the degree of freedom by a constant factor. Since the $a_{k j} w_{j}$ data can be regarded as the ratio data, we consider that it is natural to use the geometric mean as the averaging function in the DAP.

(2) In the dynamic framework of a DAP, $a_{i i}$ is the self-evaluation effect of $w_{i}(t)$ on $w_{i}(t+1)$ as shown in (1) through (6), and for the complete information case, it is usually set as $a_{i i}=1$, which is called " the self-identity condition." But for the incomplete information case, as shown by the (modified) Harker matrix $H$, the selfevaluation effect $h_{i i}$, given by (15), (17), or (19), takes into account not only the original $a_{i i}$ but also, $\lambda$, and $\mathrm{p}$. That is, the self-evaluation effect of item $i, h_{i i}$, increases with stationary rate $\lambda$ and the number of missing elements in the $i$-th row (when $\mathrm{p}>0$ ). 
(3) The original Harker method is valid in the sense its convergent solution coincides with that of the incomplete-information arithmetic $\operatorname{DAP}(p=1)$ only when $\lambda=1$, or $\mathrm{CI}=0$. Here, $\mathrm{CI}=\mathrm{N}\left(\lambda_{-}-1\right) /(\mathrm{N}-1)(\operatorname{see}(25) \operatorname{and}(26)$, Shinohara, 2011).

(4) The weight estimation for the incomplete information case can be handled just like the complete information case by constructing modified Harker matrix $\mathrm{H}$ and applying the $p$-th power mean DAP to the matrix H. But the stationary rate $\lambda$ is needed to construct $\mathrm{H}$. Only to obtain the estimated priority weight vector, the application of the incomplete-information DAP presented in Chapter 2 is enough.

\section{References}

Saaty, T.L. (1980). The Analytic Hierarchy Process, McGraw-Hill, New York. Harker, P.T. (1987). Alternative Modes of Questioning in the Analytic Hierarchy Process, Math. Modelling, 9, 353-360.

Shinohara, Masaaki, Keikichi Osawa,\& Ken Shinohara (2005). Flow and Potential in Logarithmic Least Squares Estimation of AHP, Proceedings of the International Symposium on the Analytic Hierarchy Process 2005,F1_3,pp.1-9, (2005)

Shinohara, Masaaki (2011). AHP EIGENVECTOR VIA DYNAMIC PROCESS OF PAIRWISE COMPARISON AND AVERAGING, Proceedings of the International Symposium on the Analytic Hierarchy Process 2011, 144_019, (2011.6.16)

Mogi, Wataru, \& Masaaki Shinohara (2011). Relationship Between Unknown Weight Substitution Method and LLS Method for Incomplete Information Pairwise Comparison Matrix(in Japanese), Proceedings of the 44th Annual Conference of College of Industrial Technology, Nihon University, 1103-1106 (2011.12.3).

Shinohara, Masaaki , \& Wataru Mogi (2012). Geometric Mean Formula and Topological Formula for LLS Solution of Incomplete Information AHP Pairwise Comparison (in Japanese), Proceedings of the 45th Annual Conference of College of Industrial Technology, Nihon University, 2-81 (2012.12.1).

Shinohara, Masaaki (2013). PATH ALGEBRAIC AHP EIGENVECTOR BASED ON MAX AND MIN OPERATORS, Proceedings of the International Symposium on the Analytic Hierarchy Process 2013,(2013.6.25) 